\title{
A FORMULA FOR A QUARTIC INTEGRAL: A SURVEY OF OLD PROOFS AND SOME NEW ONES
}

\author{
TEWODROS AMDEBERHAN AND VICTOR H. MOLL
}

\begin{abstract}
We discuss several existing proofs of the value of a quartic integral and present a new proof that evolved from rational Landen transformations. We include our personal renditions as related to the history of these particular computations.
\end{abstract}

\section{INTRODUCTION}

The evaluation of definite integrals has attracted the scientific community, both professional and amateurs, for a long time. Some of these evaluations are collected in tables. The authors' favorite one is by I. Gradshteyn and I. Ryzhik [11. It was the naive attempt to prove all the formulas in [1] that produced [6]. A recent review of this book [10] describes how R. Feynman 'once claimed that he acquired his initial reputation not as a physicist, but as a redoubtable evaluator of integrals'.

In the Fall of 1995, when the author (VHM) was teaching a graduate class in Analysis, a student (George Boros) stated that he could prove the identity

$$
\int_{0}^{\infty} \frac{d x}{\left(x^{4}+2 a x^{2}+1\right)^{m+1}}=\frac{\pi}{2} \frac{P_{m}(a)}{[2(a+1)]^{m+\frac{1}{2}}},
$$

where $P_{m}(a)$ is a polynomial in $a$. The integral above will be denoted by $N_{0,4}(a ; m)$. This was the introduction of VHM to the wonderful world of definite integrals. George's original expression for the coefficients $d_{l}(m)$ in

$$
P_{m}(a)=\sum_{l=0}^{m} d_{l}(m) a^{l}
$$

was

$d_{l}(m)=\sum_{j=0}^{l} \sum_{s=0}^{m-l} \sum_{k=s+l}^{m} \frac{(-1)^{k-l-s}}{8^{k}}\left(\begin{array}{c}2 k \\ k\end{array}\right)\left(\begin{array}{c}2 m+1 \\ 2 s+2 j\end{array}\right)\left(\begin{array}{c}m-s-j \\ m-k\end{array}\right)\left(\begin{array}{c}s+j \\ j\end{array}\right)\left(\begin{array}{c}k-s-j \\ l-j\end{array}\right)$.

Clearly not very useful. Intrigued by the fact that symbolic languages, such as Mathematica or Maple, were unable to evaluate this integral, and being unaware of any useful technique available for the computation of integrals, the second author joined George Boros in the study of this polynomial.

Date: February 11, 2013.

1991 Mathematics Subject Classification. Primary 33.

Key words and phrases. Integrals, WZ-method, Landen transformations, rational integrals. 
Computing some of these coefficients we observed that they are all positive. For example, if $m=5$, we obtain

$$
\left\{d_{l}(5): 0 \leq l \leq 5\right\}=\left\{\frac{4389}{256}, \frac{8589}{128}, \frac{7161}{64}, \frac{777}{8}, \frac{693}{16}, \frac{63}{8}\right\} .
$$

In this paper, we describe several proofs of the identity (1.1) where the coefficients $d_{l}(m)$ are given by

$$
d_{l}(m)=2^{-2 m} \sum_{k=l}^{m} 2^{k}\left(\begin{array}{c}
2 m-2 k \\
m-k
\end{array}\right)\left(\begin{array}{c}
m+k \\
m
\end{array}\right)\left(\begin{array}{l}
k \\
l
\end{array}\right)
$$

\section{A SURVEY OF PREVIOUS PROOFS}

2.1. The elementary proof. George's original idea was simple, but had profound consequences. The change of variables $x=\tan \theta$ yields

$$
N_{0,4}(a ; m)=\int_{0}^{\pi / 2}\left(\frac{\cos ^{4} \theta}{\sin ^{4} \theta+2 a \sin ^{2} \theta \cos ^{2} \theta+\cos ^{4} \theta}\right)^{m+1} \times \frac{d \theta}{\cos ^{2} \theta} .
$$

Now, we observe that the denominator of the trigonometric function in the integrand, is a polynomial in $u=2 \theta$. In detail,

$$
\sin ^{4} \theta+2 a \sin ^{2} \theta \cos ^{2} \theta+\cos ^{4} \theta=(1+a)+(1-a) \cos ^{2} u .
$$

In terms of the double-angle $u=2 \theta$, the original integral becomes

$$
N_{0,4}(a ; m)=2^{-(m+1)} \int_{0}^{\pi}\left(\frac{(1+\cos u)^{2}}{(1+a)+(1-a) \cos ^{2} u}\right)^{m+1} \times \frac{d u}{1+\cos u} .
$$

Expanding the binomial $(1+\cos u)^{2 m+1}$, the reader will check that

$$
\int_{0}^{\pi}\left[(1+a)+(1-a) \cos ^{2} u\right]^{-(m+1)} \cos ^{j} u d u=0
$$

for $j$ odd. The vanishing of half of the terms in the binomial expansion turned out to be a crucial property. The remaining integrals, those with $j$ even, can be simplified by using the double-angle trick one more time. The result is

$$
N_{0,4}(a ; m)=\sum_{j=0}^{m} 2^{-j}\left(\begin{array}{c}
2 m+1 \\
2 j
\end{array}\right) \int_{0}^{\pi}[(3+a)+(1-a) \cos v]^{-(m+1)}(1+\cos v)^{j} d v
$$

where $v=2 u$ and we have used the symmetry of cosine about $v=\pi$ to reduce the integrals form $[0,2 \pi]$ to $[0, \pi]$. The familiar change of variables $z=\tan (v / 2)$ produces the complicated formula for the coefficients $d_{l}(m)$ given in the Introduction.

Excited with this evaluation and considering that we could not find it in [1], we wrote this as a paper and submitted it for publication. The reviewer was unforgiving: Anybody who knows anything about hypergeometric functions would be able to do this. We realized that it was time to learn about hypergeometric functions. Eventually, we convinced an undergraduate at Tulane University to check the details of the evaluation discussed above. They formed part of her final Honor Project and the results appeared in [7]. 
2.2. The hypergeometric proof. The integral $N_{0,4}(a ; m)$ can not be found in the compendium [11] in its original form. Naturally, it is possible that it appears in some disguised form, after a change of variables. In this case, the simplest change is $t=x^{2}$, that produces

$$
N_{0,4}(a ; m)=\frac{1}{2} \int_{0}^{\infty} \frac{t^{-1 / 2} d t}{\left(t^{2}+2 a t+1\right)^{m+1}} .
$$

Integrals of this type are listed in section 3.252 of [11. Indeed, 3.252.11 states that

$$
\int_{0}^{\infty} \frac{t^{\nu-1} d t}{\left(t^{2}+2 a t+1\right)^{\mu+1 / 2}}=2^{-\mu}\left(a^{2}-1\right)^{\mu / 2} \Gamma(1-\mu) B(\nu-2 \mu+1,-\nu) P_{\nu-\mu}^{\mu}(a) .
$$

The special functions involved here are the gamma function defined by

$$
\Gamma(s)=\int_{0}^{\infty} t^{s-1} e^{-t} d t,
$$

that the reader must have seen as a generalization of integer factorials (namely that $\Gamma(n)=(n-1) !)$, and the beta function defined by

$$
B(x, y)=\int_{0}^{1} t^{x-1}(1-t)^{y-1} d t,
$$

that appears in beginning courses in Statistics, and finally the associated Legendre function $P_{\nu}^{\mu}(z)$ which was not very familiar to us. The table [11] is a good first source for special functions; in particular, sections 8.7 and 8.8 are dedicated to $P_{\nu}^{\mu}(z)$, where one learns that these functions are solutions of the differential equation

$$
\left(1-z^{2}\right) \frac{d^{2} y}{d z^{2}}-2 z \frac{d y}{d z}+\left[\nu(\nu+1)-\frac{\mu^{2}}{1-z^{2}}\right] y=0 .
$$

At the beginning of Section 8 in [11, one finds the formula 8.702:

$$
P_{\nu}^{\mu}(z)=\frac{1}{\Gamma(1-\mu)}\left(\frac{z+1}{z-1}\right)^{\mu / 2}{ }_{2} F_{1}\left(-\nu, \nu+1 ; 1-\mu ; \frac{1-z}{2}\right) .
$$

The hypergeometric function ${ }_{2} F_{1}(a, b ; c ; z)$ encountered above, is defined as the series

$$
{ }_{2} F_{1}(a, b ; c ; z):=\sum_{k=0}^{\infty} \frac{(a)_{k}(b)_{k}}{(c)_{k} k !} z^{k}
$$

where

$$
(a)_{k}=a(a+1)(a+2) \cdots(a+k-1)
$$

is the Pochhammer symbol. This function includes most of the elementary functions. For example,

$$
(1+z)^{n}={ }_{2} F_{1}(-n, b ; b ;-z), \quad \text { for any } b,
$$

and

$$
\ln z=(z-1){ }_{2} F_{1}(1,1 ; 2,1-z)
$$

just to name a couple. Section 9.121 of [1] contain many more.

Using these identities in (1.1) produces

$$
N_{0,4}(a ; m)=2^{m-1 / 2}(a+1)^{-(m+1 / 2)} B\left(2 m+\frac{3}{2}, \frac{1}{2}\right){ }_{2} F_{1}\left[-m, m+1 ; m+\frac{3}{2} ; \frac{1-a}{2}\right] \text {, }
$$


and the formula (1.4) follows from here. The details appear in [2]. The reviewer of [7] was correct: not much of the hypergeometric world is required to prove this.

2.3. A detour into the world of Ramanujan. Not knowing about hypergeometric functions and before we search for this type of proof, we observed that

$$
\int_{0}^{\infty} \frac{d x}{b x^{4}+2 a x^{2}+1}=\frac{\pi}{2 \sqrt{2}} \frac{1}{\sqrt{a+\sqrt{b}}}
$$

From here one sees that if we define

$$
g(c):=\int_{0}^{\infty} \frac{d x}{x^{4}+2 a x^{2}+1+c}
$$

and

$$
h(c)=\sqrt{a+\sqrt{1+c}},
$$

then (2.11) shows that $g(c)=\pi \sqrt{2} h^{\prime}(c)$. In particular,

$$
h^{\prime}(0)=\frac{1}{\pi \sqrt{2}} N_{0,4}(a ; 0) .
$$

Now it is clear how to proceed: further differentiation yields

$$
\sqrt{a+\sqrt{1+c}}=\sqrt{a+1}+\frac{1}{\pi \sqrt{2}} \sum_{k=1}^{\infty} \frac{(-1)^{k-1}}{k} N_{0,4}(a ; k-1) c^{k} .
$$

Using Ramanujan Master's Theorem [1, we were able to establish the identity

$$
\int_{0}^{\infty} \frac{x^{m-1} d x}{(a+\sqrt{1+x})^{2 m+1 / 2}}=\frac{1}{\pi} 2^{6 m+3 / 2}\left[m\left(\begin{array}{c}
4 m \\
2 m
\end{array}\right)\left(\begin{array}{c}
2 m \\
m
\end{array}\right)\right]^{-1} \times N_{0,4}(a ; m) .
$$

The integral on the left can be evaluated in elementary form to produce (1.4). The details appear in 4. It was never clear to the second author, how George thought of using Ramanujan's Master Theorem. He simply knew how to integrate.

2.4. Another elementary proof. The elementary proof described below was shown to us by an (anonymous) referee. Write (2.14) as

$$
\int_{0}^{\infty} \frac{d x}{x^{4}+2(b-1) x^{2}+1+c}=\pi \sqrt{2} f^{\prime}(c)
$$

with

$$
f(c)=\sqrt{b+(\sqrt{1+c}-1)} .
$$

Differentiating $m$ times with respect to $c$ yields

$$
m !(-1)^{m} \int_{0}^{\infty} \frac{d x}{\left(x^{4}+2(b-1) x^{2}+1+c\right)^{m+1}}=\pi \sqrt{2} f^{(m+1)}(c) .
$$

Hence

$$
N_{0,4}(b-1 ; m)=\frac{\pi \sqrt{2}}{m !}(-1)^{m} f^{(m+1)}(0)
$$

But

$$
f(c)=\sqrt{b}\left(1+\frac{\sqrt{1+c}-1}{b}\right)^{1 / 2}=\sqrt{b} \sum_{n=0}^{\infty}\left(\begin{array}{c}
\frac{1}{2} \\
n
\end{array}\right) b^{-n}(\sqrt{1+c}-1)^{n} .
$$


Now use the expansion

$$
(\sqrt{1+c}-1)^{n}=\left(\frac{c}{2}\right)^{n}\left\{1+n \sum_{i=1}^{\infty} \frac{(-1)^{i}(n+2 i-1) !}{i !(n+i) !}\left(\frac{c}{4}\right)^{i}\right\},
$$

given in [11, Formula 1.114, and the identity

$$
\left(\begin{array}{c}
\frac{1}{2} \\
n
\end{array}\right)=\frac{(-1)^{n-1}}{n 2^{2 n-1}}\left(\begin{array}{c}
2 n-2 \\
n-1
\end{array}\right)
$$

and taking the coefficient of $c^{m+1}$ to obtain

$$
N_{0,4}(a ; m)=\frac{\pi}{(8 a+8)^{m+1 / 2}} \sum_{i=0}^{m}\left(\begin{array}{c}
2 m-2 i \\
k-i
\end{array}\right)\left(\begin{array}{c}
m+i \\
m
\end{array}\right) 2^{i}(a+1)^{i},
$$

as desired.

\section{A Change of VARIAbles AND THE NEW PROOF}

It soon became clear that the change of variables

$$
y=R_{2}(x):=\frac{x^{2}-1}{2 x}
$$

was the key to extend the elementary method to integrals of higher order. The inverse has two branches

$$
x=y \pm \sqrt{y^{2}+1},
$$

where the plus sign is valid for $x \in[0,+\infty)$ and the other one on $(-\infty, 0]$. The rational function $R_{2}$ arises from the identity

$$
\cot 2 \theta=R_{2}(\cot \theta) .
$$

This change of variables gives the proof of the next theorem and it leads to interesting equality among integrals.

Theorem 3.1. Let $f$ be a rational function and assume that the integral of $f$ over $\mathbb{R}$ is finite. Then

$$
\begin{aligned}
\int_{-\infty}^{\infty} f(x) d x & =\int_{-\infty}^{\infty}\left[f\left(y+\sqrt{y^{2}+1}\right)+f\left(y-\sqrt{y^{2}+1}\right)\right] d y+ \\
& +\int_{-\infty}^{\infty}\left[f\left(y+\sqrt{y^{2}+1}\right)-f\left(y-\sqrt{y^{2}+1}\right)\right] \frac{y d y}{\sqrt{y^{2}+1}}
\end{aligned}
$$

Moreover, if $f$ is an even rational function, the identity (3.4) remains valid by replacing the interval of integration from $\mathbb{R}$ to $\mathbb{R}^{+}$.

The reader will check that the integrand on the right hand side of (3.4) is also a rational function. This new function is an even rational function if $f$ is such.

The first application of Theorem 3.1] was given in [3] where we proved that the integral

$$
U_{6}(a, b ; c, d, e):=\int_{0}^{\infty} \frac{c x^{4}+d x^{2}+e}{x^{6}+a x^{4}+b x^{2}+1} d x
$$


is invariant under the change of parameters

$$
\begin{aligned}
a & \rightarrow \frac{a b+5 a+5 b+9}{(a+b+2)^{4 / 3}} \\
b & \rightarrow \frac{a+b+6}{(a+b+2)^{2 / 3}} \\
c & \rightarrow \frac{c+d+e}{(a+b+2)^{2 / 3}} \\
d & \rightarrow \frac{c(b+2)+2 d+e(a+3)}{a+b+2} \\
e & \rightarrow \frac{c+e}{(a+b+2)^{1 / 3}} .
\end{aligned}
$$

Iteration of (3.6) produces a sequence of parameters $\left(a_{n}, b_{n} ; c_{n}, d_{n}, e_{n}\right)$ with

$$
U_{6}\left(a_{n}, b_{n} ; c_{n}, d_{n}, e_{n}\right)=U_{6}(a, b ; c, d, e) .
$$

Moreover, as $n \rightarrow \infty$, we have that $a_{n}, b_{n} \rightarrow 3$ and there exists a number $L$ such that $\left(c_{n}, d_{n}, e_{n}\right) \rightarrow L(1,2,1)$. Passing to the limit in (3.7) we obtain

$$
U_{6}(a, b ; c, d, e)=\frac{\pi}{2} L .
$$

This is precisely the rational function analog of the transformation of parameters

$$
\begin{aligned}
a & \mapsto \frac{a+b}{2} \\
b & \mapsto \sqrt{a b} .
\end{aligned}
$$

that leaves the elliptic integral

$$
G(a, b)=\int_{0}^{\pi / 2} \frac{d \varphi}{\sqrt{a^{2} \cos ^{2} \varphi+b^{2} \sin ^{2} \varphi}}
$$

invariant. In this situation, the sequence $\left(a_{n}, b_{n}\right)$ obtained by iterating (3.9) converges to a common limit: the arithmetic-geometric mean of $a$ and $b$, denoted by $\operatorname{AGM}(a, b)$. In the limit, we have

$$
G(a, b)=\frac{\pi}{2 \operatorname{AGM}(a, b)},
$$

precisely as in (3.8). Information about the AGM can be found in [8] and [9].

This was the beginning of a series of results on changes of parameters that leave integrals of rational functions invariant. We have called (3.6) a rational Landen transformation. These were extended to all even rational functions in [5] and the convergence of the iterations of these transformation were described in [13]. The extension to arbitrary rational functions on the whole line is described in [14] and [15]. It is an open question to produce rational Landen transformations for a general rational function on the interval $[0, \infty)$. The vanishing of the integrals such as (2.1) does not happen if the integrand is not an even rational function.

From the point of view of numerical calculations, the rational Landen transformations give a procedure to evaluate definite integrals in iterative form. For example, in the simplest case of the elementary integral

$$
I=\int_{-\infty}^{\infty} \frac{d x}{a x^{2}+b x+c}
$$


with $b^{2}-4 a c<0$, we have shown in [15] that $I$ is invariant under the change of parameters

$$
\begin{aligned}
a_{n+1} & =a_{n}\left[\frac{\left(a_{n}+3 c_{n}\right)^{2}-3 b_{n}^{2}}{\left(3 a_{n}+c_{n}\right)\left(a_{n}+3 c_{n}\right)-b_{n}^{2}}\right], \\
b_{n+1} & =b_{n}\left[\frac{3\left(a_{n}-c_{n}\right)^{2}-b_{n}^{2}}{\left(3 a_{n}+c_{n}\right)\left(a_{n}+3 c_{n}\right)-b_{n}^{2}}\right], \\
c_{n+1} & =c_{n}\left[\frac{\left(3 a_{n}+c_{n}\right)^{2}-3 b_{n}^{2}}{\left(3 a_{n}+c_{n}\right)\left(a_{n}+3 c_{n}\right)-b_{n}^{2}}\right] .
\end{aligned}
$$

Moreover, the error defined by

$$
e_{n}=\left(a_{n}-\frac{1}{2} \sqrt{4 a c-b^{2}}, b_{n}, c_{n}-\frac{1}{2} \sqrt{4 a c-b^{2}}\right),
$$

decays to 0 at a cubic rate, that is, $\left\|e_{n+1}\right\| \leq C\left\|e_{n}\right\|^{3}$.

The transformation (3.1) and its higher order analogues, given by the rational function $R_{m}$ defined by

$$
\cot (m \theta)=R_{m}(\cot \theta),
$$

have produced interesting results for definite integrals of higher degree. See [14 for details.

The second goal of this note is to use the Landen transformation (3.1) to produce a new proof of the identity (1.1) and thus obtain the more explicit natural form of the coefficients $d_{l}(m)$ given in (1.4).

Theorem 3.2. For $m \in \mathbb{N}$, let

$$
Q(x)=\frac{1}{\left(x^{4}+2 a x^{2}+1\right)^{m+1}} .
$$

Define

$$
\begin{aligned}
Q_{1}(y) & :=\left[Q\left(y+\sqrt{y^{2}+1}\right)+Q\left(y-\sqrt{y^{2}+1}\right)\right]+ \\
& +\frac{y}{\sqrt{y^{2}+1}}\left[Q\left(y+\sqrt{y^{2}+1}\right)-Q\left(y-\sqrt{y^{2}+1}\right)\right] .
\end{aligned}
$$

Then

$$
Q_{1}(y)=\frac{T_{m}(2 y)}{2^{m}\left(1+a+2 y^{2}\right)^{m+1}},
$$

where

$$
T_{m}(y)=\sum_{k=0}^{m}\left(\begin{array}{l}
m+k \\
m-k
\end{array}\right) y^{2 k} .
$$

Proof. Introduce the variable $\phi=y+\sqrt{y^{2}+1}$. Then $y-\sqrt{y^{2}+1}=-\phi^{-1}$ and $y=\phi-\phi^{-1}$. Moreover,

$$
\begin{aligned}
Q_{1}(y) & =\left[Q(\phi)+Q\left(\phi^{-1}\right)\right]+\frac{\phi^{2}-1}{\phi^{2}+1}\left(Q(\phi)-Q\left(\phi^{-1}\right)\right) \\
& =\frac{2}{\phi^{2}+1}\left[\phi^{2} Q(\phi)+Q\left(\phi^{-1}\right)\right] \\
& :=S_{m}(\phi) .
\end{aligned}
$$


The result of the theorem is equivalent to

$$
\left.2^{m}\left(1+a+\frac{1}{2}\left(\phi-\phi^{-1}\right)^{2}\right)\right)^{m+1} S_{m}(\phi)=T_{m}\left(\phi-\phi^{-1}\right) .
$$

A direct simplification of the left hand side of (3.19) shows that this identity is equivalent to proving

$$
\frac{\phi^{2 m+1}+\phi^{-(2 m+1)}}{\phi+\phi^{-1}}=T_{m}\left(\phi-\phi^{-1}\right) .
$$

First proof. One simply checks that both sides of (3.20) satisfy the second order recurrence

$$
c_{m+2}-\left(\phi^{2}+\phi^{-2}\right) c_{m+1}+c_{m}=0,
$$

and the values for $m=0$ and $m=1$ match. This is straight-forward for the expression on the left hand side, while the WZ-method settles the right hand side.

Note. The WZ-method is an algorithm in Computational Algebra that, among other things, will produce for a hypergeometric/holonomic sum, such as (3.18), a recurrence like (3.21). The reader will find in [17] and [16] information about this algorithm.

Second proof. In [12, one finds the generating function

$$
B_{t}(z)=\sum_{k \geq 0}(t k)_{k-1} \frac{z^{k}}{k !}
$$

where $(a)_{k}=a(a+1) \ldots(a+k-1)$ is the Pochhammer symbol. The special values

$$
B_{-1}(z)=\frac{1+\sqrt{1+4 z}}{2}, \quad \text { and } B_{2}(z)=\frac{1-\sqrt{1-4 z}}{2 z},
$$

are combined to produce the identity

$$
\frac{1}{\sqrt{1+4 z}}\left(B_{-1}(z)^{n+1}-(-z)^{n+1} B_{2}(-z)^{n+1}\right)=\sum_{k=0}^{n}\left(\begin{array}{c}
n-k \\
k
\end{array}\right) z^{k} .
$$

Replace $n$ by $2 m$ and $z$ by $\left(4 y^{2}\right)^{-1}$ to produce

$$
\frac{1}{2 \sqrt{1+y^{2}}(2 y)^{2 m}}\left(\phi^{2 m+1}+\phi^{-(2 m+1)}\right)=\sum_{k=0}^{m}\left(\begin{array}{c}
2 m-k \\
k
\end{array}\right) z^{k} .
$$

We also have

$$
\begin{aligned}
T_{m}(y) & =\sum_{k=0}^{m}\left(\begin{array}{c}
m+k \\
m-k
\end{array}\right) y^{2 k} \\
& =y^{2 m} \sum_{k=0}^{m}\left(\begin{array}{c}
2 m-k \\
k
\end{array}\right) y^{-2 k}
\end{aligned}
$$

Thus,

$$
\begin{aligned}
T_{m}\left(\phi-\phi^{-1}\right) & =T_{m}(2 y) \\
& =(2 y)^{2 m} \sum_{k=0}^{m}\left(\begin{array}{c}
2 m-k \\
k
\end{array}\right) z^{k} .
\end{aligned}
$$


We conclude that

$$
T_{m}\left(\phi-\phi^{-1}\right)=\frac{1}{2 \sqrt{1+y^{2}}}\left(\phi^{2 m+1}+\phi^{-(2 m+1)}\right),
$$

and the result follows from $\phi+\phi^{-1}=2 \sqrt{y^{2}+1}$.

We now prove (1.1). The identity in Theorem 3.1 shows that

$$
\int_{0}^{\infty} Q(x) d x=\int_{0}^{\infty} Q_{1}(y) d y
$$

and this last integral can be evaluated in elementary terms. Indeed,

$$
\begin{aligned}
\int_{0}^{\infty} Q_{1}(y) d y & =\int_{0}^{\infty} \frac{T_{m}(2 y) d y}{2^{m}\left(1+2 y^{2}\right)^{m+1}} \\
& =\frac{1}{2^{m}} \sum_{k=0}^{m}\left(\begin{array}{c}
m+k \\
m-k
\end{array}\right) \int_{0}^{\infty} \frac{(2 y)^{2 k} d y}{\left(1+a+2 y^{2}\right)^{m+1}}
\end{aligned}
$$

The change of variables $y=\frac{\sqrt{1+a}}{\sqrt{2}} t$ gives

$$
\int_{0}^{\infty} Q_{1}(y) d y=\frac{1}{[2(1+a)]^{m+1 / 2}} \sum_{k=0}^{m}\left(\begin{array}{c}
m+k \\
m-k
\end{array}\right) 2^{k}(1+a)^{k} \int_{0}^{\infty} \frac{t^{2 k} d t}{\left(1+t^{2}\right)^{m+1}},
$$

and the elementary identity

$$
\int_{0}^{\infty} \frac{t^{2 k} d t}{\left(1+t^{2}\right)^{m+1}}=\frac{\pi}{2^{2 m+1}}\left(\begin{array}{c}
2 k \\
k
\end{array}\right)\left(\begin{array}{c}
2 m-2 k \\
m-k
\end{array}\right)\left(\begin{array}{c}
m \\
k
\end{array}\right)^{-1}
$$

gives

$$
\int_{0}^{\infty} Q_{1}(y) d y=\frac{\pi}{2^{2 m+1}} \frac{1}{[2(1+a)]^{m+1 / 2}} \sum_{k=0}^{m}\left(\begin{array}{c}
m+k \\
m-k
\end{array}\right) 2^{k}\left(\begin{array}{c}
2 k \\
k
\end{array}\right)\left(\begin{array}{c}
2 m-2 k \\
m-k
\end{array}\right)\left(\begin{array}{c}
m \\
k
\end{array}\right)^{-1}(1+a)^{k} .
$$

This can be simplified further using

$$
\left(\begin{array}{c}
m+k \\
m-k
\end{array}\right)\left(\begin{array}{c}
2 k \\
k
\end{array}\right)=\left(\begin{array}{c}
m+k \\
m
\end{array}\right)\left(\begin{array}{c}
m \\
k
\end{array}\right)
$$

and the equality (3.26) to produce

$$
\int_{0}^{\infty} Q(y) d y=\frac{\pi}{2^{2 m+1}} \frac{1}{[2(1+a)]^{m+1 / 2}} \sum_{k=0}^{m} 2^{k}\left(\begin{array}{c}
m+k \\
m
\end{array}\right)\left(\begin{array}{c}
2 m-2 k \\
m-k
\end{array}\right)(1+a)^{k} .
$$

This completes the proof of (1.1), with the coefficients $d_{l}(m)$ given in (1.4).

Acknowledgments. The work of the second author was partially funded by NSF-DMS 0409968. 


\section{REFERENCES}

[1] B. Berndt. Ramanujan's Notebooks, Part I. Springer-Verlag, New York, 1985.

[2] G. Boros and V. Moll. An integral hidden in Gradshteyn and Ryzhik. Jour. Comp. Applied Math., 106:361-368, 1999.

[3] G. Boros and V. Moll. A rational Landen transformation. The case of degree 6. In Knopp G. Mendoza E.T. Quinto E. L. Grinberg S. Berhanu M, editor, Contemporay Mathematics. Analysis, Geometry, Number Theory: The Mathematics of Leon Ehrenpreis, volume 251, pages 83-89. American Mathematical Society, 2000.

[4] G. Boros and V. Moll. The double square root, Jacobi polynomials and Ramanujan's master theorem. Jour. Comp. Applied Math., 130:337-344, 2001.

[5] G. Boros and V. Moll. Landen transformation and the integration of rational functions. Math. Comp., 71:649-668, 2001.

[6] G. Boros and V. Moll. Irresistible Integrals. Cambridge University Press, New York, 1st edition, 2004.

[7] G. Boros, V. Moll, and S. Riley. An elementary evaluation of a quartic integral. Scientia, 11:1-12, 2005.

[8] J. M. Borwein and P. B. Borwein. Pi and the AGM- A study in analytic number theory and computational complexity. Wiley, New York, 1st edition, 1987.

[9] D. Cox. Gauss and the Arithmetic-Geometric mean. Notices AMS, 32:147-151, 1985.

[10] J. J. Foncannon. Review of Irresistible Integrals: Symbolics, Analysis and Experiments in the Evaluation of Integrals, by George Boros and Victor H. Moll. Math. Intelligencer, 28:65-68, 2006.

[11] I. S. Gradshteyn and I. M. Ryzhik. Table of Integrals, Series, and Products. Edited by A. Jeffrey and D. Zwillinger. Academic Press, New York, 6th edition, 2000.

[12] R. Graham, D. Knuth, and O. Patashnik. Concrete Mathematics. Addison Wesley, Boston, 2nd edition, 1994.

[13] J. Hubbard and V. Moll. A geometric view of rational Landen transformation. Bull. London Math. Soc., 35:293-301, 2003.

[14] D. Manna and V. Moll. Rational Landen transformations on R. Math. Comp., 2007.

[15] D. Manna and V. Moll. A simple example of a new class of Landen transformations. Amer. Math. Monthly, 114:232-241, 2007.

[16] I. Nemes, M. Petkovsek, H. Wilf, and D. Zeilberger. How to do MONTHLY problems with your computer. Amer. Math. Monthly, 104:505-519, 1997.

[17] M. Petkovsek, H. Wilf, and D. Zeilberger. $A=B$. A. K. Peters, Ltd., 1st edition, 1996.

Department of Mathematics, Tulane University, New Orleans, LA 70118

E-mail address: tamdeberhan@math.tulane.edu

Department of Mathematics, Tulane University, New Orleans, LA 70118

E-mail address: vhm@math.tulane.edu 